\title{
Article
}

\section{Influences of Linkage Stiffness on Rupture Rate in Single-Molecule Pulling Experiments} Han-Jou Lin, Yu-Jane Sheng, Hsuan-Yi Chen, and Heng-Kwong Tsao

J. Phys. Chem. B, 2007, 111 (23), 6493-6500 • DOI: 10.1021/jp070881x

Downloaded from http://pubs.acs.org on November 24, 2008

\section{More About This Article}

Additional resources and features associated with this article are available within the HTML version:

- $\quad$ Supporting Information

- $\quad$ Access to high resolution figures

- $\quad$ Links to articles and content related to this article

- $\quad$ Copyright permission to reproduce figures and/or text from this article

\section{View the Full Text HTML}

\section{ACS Publications}




\title{
Influences of Linkage Stiffness on Rupture Rate in Single-Molecule Pulling Experiments
}

\author{
Han-Jou Lin, ${ }^{\dagger}$ Yu-Jane Sheng, ${ }^{*} \dagger$ Hsuan-Yi Chen,,${ }^{\ddagger}$ and Heng-Kwong Tsao $*, \S$ \\ Department of Chemical Engineering, National Taiwan University, Taipei, Taiwan 106, R.O.C., Department of \\ Physics, Graduate Institute of Biophysics, and Center for Complex Systems, National Central University, \\ Jhongli, Taiwan 320, R.O.C., Department of Chemical and Materials Engineering, National Central University, \\ Jhongli, Taiwan 320, R.O.C.
}

Received: February 1, 2007; In Final Form: April 6, 2007

\begin{abstract}
In the dissociation of a noncovalent biomolecular bond by external pulling, the bonded site is often connected to the force-acting site by a linkage. The role of the linkage stiffness on the rupture of a ligand-receptor complex under constant force is investigated by overdamped Langevin dynamics for the elastically coupled ligand and probe. The effects on the bond lifetime include effective ligand diffusivity, force fluctuations, and violation of adiabatic condition. The rupture rate declines with increasing linkage stiffness. For soft linkage, the effect associated with the spring and probe can be ignored, and the true rupture rate can be extracted. On the other hand, for stiff linkage, the diffusivity of the probe has to be accounted for and, thus, leads to a lower rupture rate, depending on the diffusivity ratio between the probe and ligand. Nevertheless, the energy barrier height can be reasonably extracted by constant pulling experiments, regardless of the linkage stiffness.
\end{abstract}

\section{Introduction}

Noncovalent biomolecular bonds, such as receptor-ligand interactions, mediate many of life's functions in cells. They are also very important in applications such as biosensors and biomaterials. Recently, the physical nature of weak noncovalent bonds has been experimentally explored at the single-molecule level. ${ }^{1,2}$ In these experiments, such as protein unfolding and ligand-receptor dissociation, the externally applied force (in the piconewton range) is exerted on an anchored molecular complex. Most direct measurements of single bond strength have been conducted with three types of ultrasensitive force-probing techniques: atomic force microscope (AFM), biomembrane force probe, and laser optical tweezer. Typically, the pulling spring is moved away from the anchored molecule at a constant velocity. The force is, therefore, slowly increased until bond rupture occurs. In constrast to equilibrium binding properties, the bond-rupture force, adopted in depicting bond strength, is not constant but, instead, is dependent upon the rate of force increment (loading rate).

The fact that the natural lifetime of a ligand-receptor complex is long (millisecond to second) reveals the existence of an energy barrier, which must be crossed by the thermally activated escape (unbinding at zero force). The lifetime is stochastic in nature due to kinetic escape. Thus, the unbinding process of the bound complex along the reaction coordinate on the free energy landscape can be considered the escape of an overdamped particle from a kinetic trap. ${ }^{3-7}$ When an external force $(F)$ is applied, the kinetic escape across the barrier is assisted mechanically, and thereby, the escape time is reduced. In fact, the main goal of performing single-molecule pulling

* To whom correspondence should be addressed. E-mail: yjsheng@ ntu.edu.tw and hktsao@cc.ncu.edu.tw.

National Taiwan University.

$\doteqdot$ Department of Physics, Graduate Institute of Biophysics, and Center for Complex Systems, National Central University.

$\S$ Department of Chemical and Materials Engineering, National Central University. experiments is to extract the intrinsic kinetic parameters, including the reaction range $(a)$, the energy barrier $\left(E_{a}\right)$, and the rate constant $(k)$. Owing to the pulling, the critical force $\left(F_{\mathrm{c}}\right)$, which represents the maximum slope of the trapped potential, also plays an important role in determining the rupture rate. When pulling is absent $(F=0)$, the bound complex dissociates eventually. This thermally activated escape characterizes the kinetic limit. On the contrary, as $F>F_{\mathrm{c}}$, the dissociation is dominated by mechanical pulling. Under constant pulling, $F<F_{\mathrm{c}}$, the free-energy surface is altered and the energy barrier is lowered. As a result, the mean first passage time (rupture time) of the forced escape from a kinetic trap decreases with increasing $F$.

Under any level of pulling, a given bond will break eventually if the force is applied over a sufficient time period. When the applied pulling is ramped up with time, the interplay between kinetic escape and mechanical pulling leads to loading-ratedependent behavior of the bond-rupture force $\left(F_{\mathrm{u}}\right) .^{3-7}$ When the loading rate $\left(F_{\mathrm{t}}\right)$ is very slow, the time is long enough so that the rupture occurs mainly by thermal fluctuations, and low rupture forces result. On the other hand, if the loading rate is very fast, then no time is available for accumulated thermal actions. The rupture force therefore approaches the maximum, intrinsic binding force $\left(F_{\mathrm{c}}\right)$. Evidently, the rupture force is not an intrinsic property associated with the bound complex, and extracting useful information from pulling experiments under steady speed detachment requires an appropriate relation between the mean rupture force and the loading rate, that is, $F_{\mathrm{u}}\left(F_{\mathrm{t}}\right)$, which must involve kinetic properties, as well. Consequently, the rate constant (bond-rupture time) under constant force conditions, that is, $k(F)$, instead of bond-rupture force under constant loading rate conditions, is more direct and desirable for extracting kinetic information. In fact, from the theoretical viewpoint, $F_{\mathrm{u}}\left(F_{\mathrm{t}}\right)$ can be derived only on the basis of knowing $k(F)$.

In the dissociation or detachment events, the externally applied force is usually not acting on the bonded region directly. 


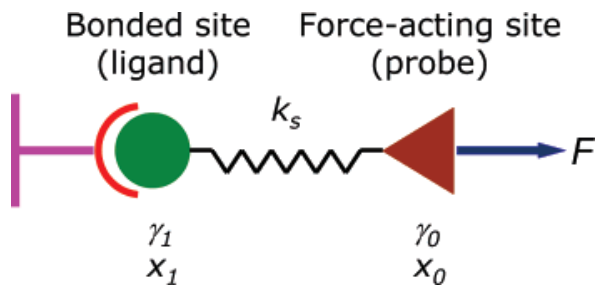

Figure 1. Schematic representation of the single-molecule pulling experiment. The ligand, which forms a noncovalent biomolecular bond with the receptor, is connected to the probe through an elastic linkage. The probe is under a constant pulling, $F$. The position and friction coefficient of the ligand and probe are $\left\{x_{1}, \gamma_{1}\right\}$ and $\left\{x_{0}, \gamma_{0}\right\}$, respectively.

In general, the bonded site is connected to another site where the force can be acting on, as shown in Figure 1. For example, in AFM experiments, the force is applied by a soft cantilever coupled to the ligand-receptor complex by a polymer. ${ }^{8,9}$ Typically, the cantilever beam and tip of the AFM were modeled as an elastic spring and a microparticle. ${ }^{7}$ Brownian effects of cantilevers in fluids have long been a source of noise contamination in AFM measurements. ${ }^{10}$ These facts disclose that both the bonded and force-acting sites are subjected to thermal fluctuations, and the stiffness of the linkage between these two sites may play an important role in determining the rupture rate associated with the single-molecule pulling experiments. Since long, flexible linkers are often used in most experiments to extract kinetic parameters, one would wonder what happens to the dissociation rate if the linker is absent or short, as in biological environments.

In a real unbinding experiment, the connection between the bonded and force-acting sites may be complicated. In order to grasp the essential features of the influence of the linkage stiffness on the rupture rate, we consider the simplest scenario in this paper: the constant pulling force is acting on the bonded object (e.g., ligand + AFM), which is represented by two overdamped particles with different friction coefficients connected by a Hookean linkage. On the basis of Brownian dynamics, we will show that the stiffness of the spring alters the rupture rate, which depends on the ratio of friction coefficients, as well. Even under the condition of constant pulling, the elastic spring may lead to significant fluctuations of the pulling force, which actually help the escape of the ligand from the binding of the receptor. A simple theory based on the Kramers rate theory ${ }^{11}$ is also given to explain the simulation results.

\section{Brownian Dynamics for a Weak-Bond Rupture}

The force-assisted rupture process of the bound complex along the reaction coordinate $x$ on the free energy landscape is equivalent to the escape of an overdampled particle from a kinetic trap, $U(x)$, with range $a$ under a pulling force. Since the force is not directly acting on the bonded site (e.g., ligand) in single-molecule pulling experiments, there exists a linkage connecting the bonded site to the force-acting site (e.g., probe), which is under constant pulling. Assume that the force acting on the probe is delivered to the ligand through an elastic linkage with spring constant, $k_{\mathrm{s}}$. As a result, the rupture of the weak bond is modeled as the escape of the two overdamped particles connected by a Hookean linkage, as illustrated in Figure 1. The first overdamped particle, with friction coefficient $\gamma_{1}$, is trapped in the energy well and subject to the external force indirectly. The second overdamped particle, with friction coefficient $\gamma_{0}$, is under a constant pulling force, $F$, which is delivered through the connected spring to the bonded particle. Since the response of the probe (spring) to the thermal fluctuation kicking the ligand cannot be instant, the dynamics of the probe must be taken into account. The overdamped Langevin equations for both ligand $\left(x_{1}\right)$ and probe $\left(x_{0}\right)$ are given by

$$
\gamma_{1} \frac{\mathrm{d} x_{1}}{\mathrm{~d} t}=-\frac{\partial U}{\partial x_{1}}+k_{\mathrm{s}}\left(x_{0}-x_{1}-l\right)+R_{1}(t)
$$

and

$$
\gamma_{0} \frac{\mathrm{d} x_{0}}{\mathrm{~d} t}=F-k_{\mathrm{s}}\left(x_{0}-x_{1}-l\right)+R_{0}(t)
$$

where $l$ is the intrinsic length of the spring. The effect of the thermal fluctuations is represented by the random force, $R_{i}(t)$, which obeys the fluctuation-dissipation theorem,

$$
\left\langle R_{i}(t) R_{i}\left(t^{\prime}\right)\right\rangle=2 \gamma_{i} k_{\mathrm{B}} T \delta\left(t-t^{\prime}\right)
$$

The friction coefficient, $\gamma_{i}$, relates to the Brownian diffusivity, $D_{i}$, by

$$
D_{i}=\frac{k_{\mathrm{B}} T}{\gamma_{i}}
$$

The dimensionless form of the overdamped Langevin equations are written as

$$
\hat{x}_{1}^{\prime}=\hat{x}_{1}+\Delta \hat{t}\left[-\frac{\partial \hat{U}}{\partial \hat{x}_{1}}+\left(\frac{k_{\mathrm{s}} a^{2}}{k_{\mathrm{B}} T}\right)\left(\hat{x}_{0}-\hat{x}_{1}-\hat{l}\right)\right]+\sqrt{2 \Delta \hat{t}} Z_{1}
$$

and

$$
\begin{array}{r}
\hat{x}_{0}^{\prime}=\hat{x}_{0}+\left(\frac{D_{0}}{D_{1}}\right) \Delta \hat{t}\left[\hat{F}-\left(\frac{k_{\mathrm{s}} a^{2}}{k_{\mathrm{B}} T}\right)\left(\hat{x}_{0}-\hat{x}_{1}-\hat{l}\right)\right]+ \\
\sqrt{2\left(\frac{D_{0}}{D_{1}}\right) \Delta \hat{t} Z_{2}}
\end{array}
$$

where $Z_{i}$ denotes a Gaussian distribution with zero mean and unit variance. The position, time, and force are scaled by $a$, $a^{2} / D_{1}$, and $k_{\mathrm{B}} T / a$, respectively. If the probe is large compared to the ligand, then $D_{0} / D_{1} \ll 1$. Typically, the integration time step is $\hat{\Delta t}=10^{-4}$.

A. Asymptotic Limits: Stiff and Soft Springs. Before we embark on numerical simulations, it is helpful to analyze the limiting behavior associated with linkage stiffness. In the present model system, there are a few characteristic length scales: the range of the binding interaction range, $a$; the elongation of the spring due to external force, $L=F / k_{\mathrm{s}}$; and the mean fluctuation of spring elongation due to thermal fluctuations $L^{\prime}=\left(k_{\mathrm{B}} T / k_{\mathrm{s}}\right)^{1 / 2}$. In addition, the characteristic random displacement due to thermal fluctuations is related to the time step by $\Delta z_{i}=$ $\sqrt{D_{i} \Delta t}$. By comparing these characteristic lengths, there are two asymptotic limits that can be realized conceptually: the soft and stiff springs.

For a stiff spring, both the elongation and elongation fluctuation are small compared to the binding range, $L \ll a$ and $L^{\prime} \ll a$. That is, the stiffness must satisfy the criterion, $F / a$ $\ll k_{\mathrm{S}}$ and $k_{\mathrm{B}} T / a^{2} \ll k_{\mathrm{S}}$. Since the variation of the relative position between $x_{0}$ and $x_{1}$ with time is small compared to $a$, one has $\mathrm{d} x_{0} / \mathrm{d} t \cong \mathrm{d} x_{1} / \mathrm{d} t$. Summing up eqs 1 and 2 yields

$$
\left(\gamma_{0}+\gamma_{1}\right) \frac{\mathrm{d} x_{1}}{\mathrm{~d} t}=-\frac{\partial U}{\partial x_{1}}+F+R_{\mathrm{s}}(t)
$$


where $R_{\mathrm{s}}(t)=R_{1}(t)+R_{2}(t)$ with the variance

$$
\left\langle R_{\mathrm{s}}(t) R_{\mathrm{s}}\left(t^{\prime}\right)\right\rangle=2\left(\gamma_{0}+\gamma_{1}\right) k_{\mathrm{B}} T \delta\left(t-t^{\prime}\right)
$$

This consequence indicates that when the spring is stiff, the probe and ligand can be regarded as single bonded object with friction coefficient $\gamma_{0}+\gamma_{1}$ under an external pulling $F$. The random velocity process for this assembly $R_{\mathrm{s}}(t)$ is consistent with the fluctuation-dissipation theorem.

Conversely, for soft spring, the elongation and elongation fluctuation are large, as compared to the binding range, $L \gg a$ and $L^{\prime} \gg a$. The criterion for the spring constant is then given by $F / a \gg k_{\mathrm{s}}$ and $k_{\mathrm{B}} T / a^{2} \gg k_{\mathrm{s}}$. Since the thermal fluctuation within the bonded region perturbs the relative position between $x_{0}$ and $x_{1}$ slightly, the elongation of the spring is primarily due to the external force, that is, $F \cong k_{\mathrm{s}}\left(x_{0}-x_{1}-l\right)$. The position variation of the probe $\left(x_{0}\right)$ is mainly as a result of its own Brownian motion in a harmonic well constructed by $k_{\mathrm{s}} x_{0}-F$. Substituting this result into eq 1 gives

$$
\gamma_{1} \frac{\mathrm{d} x_{1}}{\mathrm{~d} t}=-\frac{\partial U}{\partial x_{1}}+F+R_{1}(t)
$$

This equation indicates that when the spring is soft, the ligand is essentially subjected to a constant force directly, and the effect of the probe in determining the rupture rate is not significant. Equation 7 is consistent with the statement in Appendix B of reference 3: if the linker dynamics is sufficiently fast, then the system evolves on the potential of mean force. ${ }^{3}$

In order to investigate the bond-rupture kinetics by external pulling, the applied force is usually less than the critical force but greater than the thermal fluctuation, $F_{\mathrm{c}}>F>k_{\mathrm{B}} T / a$. The above analysis shows that the soft linkage $\left(k_{\mathrm{S}} a \ll k_{\mathrm{B}} T / a\right)$ leads to a weak correlation between the ligand and probe. The spring delivers the external pulling truthfully, and the bond rupture is dominated by the ligand's Brownian motion. On the other hand, the stiff linkage $\left(k_{\mathrm{s}} a \gg F\right)$ results in a strong correlation between the ligand and probe. The ligand and probe can be considered one rigid object that tries to escape from the energy well with smaller Brownian diffusivity.

The thermal fluctuation leads to the random displacement of the ligand. If the response of the spring and probe is instantaneous, then the ligand is always under the condition of constant pulling, $F$, after each time step, $\Delta t$. In the present study, the position relaxation time is always assumed to be large as compared to that of the Brownian correlation time (particle velocity correlation time). That is, no matter how stiff the spring is, the response time associated with the spring and probe is much greater than the Brownian correlation time. In fact, the latter is assumed to be 0 in the simulations. The response time of the spring force can be represented by $\tau_{\mathrm{r}}=\gamma_{\mathrm{m}} / k_{\mathrm{s}}$, where the effective friction coefficient $\gamma_{\mathrm{m}}$ will be defined later. The relaxation time, $\tau_{\mathrm{r}}$, is inversely proportional to the stiffness. If the spring is replaced by a rigid rod, then $k_{\mathrm{s}} \rightarrow \infty$ and $\tau_{\mathrm{r}} \rightarrow 0$. In this limit, the response time associated with the spring and probe is small as compared to the Brownian correlation time. Therefore, the behavior in this limit cannot be correctly captured by our Brownian dynamics.

Because the response time associated with the spring and probe is finite, the random displacement, $\Delta z_{i}$, results in fluctuations of the spring force acting on the ligand. When the spring is very stiff, we have to avoid the unreasonable situation that the change of the spring force caused by the random displacement is greater than the random force. As a consequence, the displacement $\Delta z_{1}$ must be small enough to fulfill the condition, $k_{\mathrm{s}}\left(\Delta z_{1}\right)^{2} \lesssim k_{\mathrm{B}} T$. Therefore, for a given linkage stiffness, the time step, $\Delta t$, must be small enough so that the scaled time step satisfies

$$
\left(\frac{k_{\mathrm{s}} a^{2}}{k_{\mathrm{B}} T}\right)^{-1}>\Delta \hat{t}
$$

This equation is essentially $\tau_{\mathrm{r}} \gg \Delta t$. In our simulations for large $k_{\mathrm{s}}, \Delta \hat{t}=10^{-6}$ must be adopted to obtain reasonable results.

\section{Forced Kramers Rate Theory}

The classical theory for the rupture experiments under constant pulling, Kramers rate theory, ${ }^{11}$ does not consider the effect of linkers. Since the Kramers theory provides us with insights for analyzing the influences of linkage stiffness, we review this theory briefly and obtain the analytical rate constant under constant force, $F$, for a model potential, which will be adopted for calculating the rupture rate of elastically coupled ligand and probe by overdamped Langevin dynamics.

The kinetics of bond rupture modeled by the escape of an overdamped particle from an energy well can be described by a phenomenological formalism if the adiabatic approximation is valid. The survival probability $P_{\mathrm{s}}(t)$ satisfies the first-order rate equation with a time-dependent rate constant, $k[F(t)]$.

$$
\frac{\mathrm{d} P}{\mathrm{~d} t}=-k(t) P(t)
$$

The solution is simply

$$
P_{\mathrm{s}}(t)=\exp \left[-\int_{0}^{t} k\left(t^{\prime}\right) \mathrm{d} t^{\prime}\right]
$$

The probability distribution of lifetime is $-\left(\mathrm{d} P_{\mathrm{s}} / \mathrm{d} t\right)$, and therefore, the mean bond lifetime is

$$
\langle T\rangle=\int_{0}^{\infty} t\left(-\frac{\mathrm{d} P_{\mathrm{s}}}{\mathrm{d} t}\right) \mathrm{d} t
$$

The rate constant is defined as $k=\langle T\rangle^{-1}$ and can be evaluated by Kramers theory, ${ }^{11}$

$$
k=\frac{1}{\tau} \exp \left(-\beta E_{a}\right)
$$

where $\beta=\left(k_{\mathrm{B}} T\right)^{-1}$ and

$$
\frac{1}{\tau}=\frac{\left|U^{\prime \prime}\left(x_{+}\right) U^{\prime \prime}\left(x_{-}\right)\right|^{1 / 2}}{2 \pi \gamma}
$$

$\gamma$ denotes the friction coefficient of the overdamped particle in the well. The positions $x_{-}$and $x_{+}$denote the well and the saddle point, respectively. The activation energy associated with the energy barrier is

$$
E_{a}=U\left(x_{+}\right)-U\left(x_{-}\right) \gg k_{\mathrm{B}} T
$$

When the spring force, $F_{\mathrm{s}}(t)$, is applied, the apparent trapping potential in eq 1 becomes

$$
V(x)=U(x)-F_{\mathrm{s}}(t) x
$$

As a result, the well and saddle points change with the timevarying force, and they can be determined by

$$
\frac{\partial U}{\partial x}\left[x_{ \pm}(t)\right]=F_{\mathrm{s}}(t)
$$


owing to $\partial V / \partial x=0$. The apparent barrier height then becomes

$$
E_{\mathrm{b}}(t)=V\left[x_{+}(t)\right]-V\left[x_{-}(t)\right]
$$

Although $E_{\mathrm{b}}$ and $\tau$ vary with time, the survival probability, $P_{\mathrm{s}}(t)$, still satisfies eq 9 if the Brownian particle is adjusted to the apparent potential, $V(x)$, instantaneously (adiabatic approximation). ${ }^{12}$

To obtain an analytical expression for the escape rate constant, $k$, under constant force, $F$, we consider a model potential in this study:

$$
U(x)=\frac{E_{a}^{0}}{2}\left[1-\cos \pi\left(\frac{x}{a}\right)\right], \quad 0 \leq x \leq a
$$

Straightforward algebra leads to $x_{-}=(a / \pi) \sin ^{-1}\left(\left(F / F_{\mathrm{c}}\right)\right), x_{+}$ $=a-x_{-}$, and $F_{\mathrm{c}}=\left(E_{a}^{0} / 2\right)((\pi / a))$. The intrinsic energy barrier and pre-exponential constant are given respectively by

$$
E_{a}(F)=E_{a}^{0}\left[\sqrt{1-\left(\frac{F}{F_{\mathrm{c}}}\right)^{2}}-\frac{F}{F_{\mathrm{c}}} \cos ^{-1}\left(\frac{F}{F_{\mathrm{c}}}\right)\right]
$$

and

$$
\frac{1}{\tau(F)}=\tau_{0}^{-1}\left[1-\left(\frac{F}{F_{\mathrm{c}}}\right)^{2}\right]^{1 / 2}
$$

where $\tau_{0}=(4 / \pi)\left(a^{2} / D\right)\left(\beta E_{a}^{0}\right)^{-1}$. In the weak pulling limit $F / F_{\mathrm{c}}$ $\ll 1$, one has $E_{a}=E_{a}{ }^{0}\left[1-(\pi / 2)\left(F / F_{\mathrm{c}}\right)+(1 / 2)\left(\left(F / F_{\mathrm{c}}\right)\right)^{2}\right] \approx E_{a}{ }^{0}$ $-F a$ and $(1 / \tau(F))=\tau_{0}{ }^{-1}\left[1-(1 / 2)\left(\left(F / F_{\mathrm{c}}\right)\right)^{2}\right] \approx \tau_{0}{ }^{-1}$, which is consistent with Bell's expression. ${ }^{13}$ In the strong pulling limit, $1-F / F_{\mathrm{c}} \ll 1$, one has $E_{a}=(2 \sqrt{2} / 3) E_{a}{ }^{0}\left(1-\left(F / F_{\mathrm{c}}\right)\right)^{3 / 2}$ and $(1 / \tau(F))=\sqrt{2} \tau_{0}{ }^{-1}\left(1-\left(F / F_{\mathrm{c}}\right)\right)^{1 / 2}$, which agree with Garg's form. ${ }^{14}$ This consequence confirms that Bell's and Garg's expressions are valid in different regimes. ${ }^{15}$ Note that the above analysis is for the escape of a Brownian particle from the trap under constant force.

\section{Results and Discussion}

The dissociation kinetics of a ligand-receptor complex has been experimentally explored by ultrasensitive force-probing techniques at the single-molecule level. In those experiments, the ligand is often connected to the force probe by a linkage; moreover, the force probe undergoes significant thermal fluctuations. Therefore, the bonded region is actually linked to the force-acting region by an elastic linkage, whose stiffness may affect the rupture process. By using overdamped Langevin dynamics, we investigate the escape kinetics of two elastically coupled Brownian particles from a trap, which represent the ligand and probe, respectively.

The variation of the rate constant $\left(k=\langle T\rangle^{-1}\right)$ with the external force for $\gamma_{0} / \gamma_{1}=5$ is plotted in Figure 2 for different spring constants. In this work, the barrier height of the trap is $\beta E_{a}{ }^{0}=$ 10 , and thus, the critical force, $\beta F_{\mathrm{c}} a$, is $5 \pi$. The rate constant rises with increasing $F$ for a given linkage stiffness. This is simply because the external force lowers the effective barrier height. On the other hand, the rate constant declines with increasing $k_{\mathrm{s}}$ for a specified external force. One can estimate the force-dependence rate constant by the forced Kramers rate theory, eq 17. As illustrated in Figure 2, the rate constant for the soft spring $\left(k_{\mathrm{S}} a^{2} / k_{\mathrm{B}} T=10^{-1}\right)$ can be reasonably represented by the Kramers theory with $\gamma_{1}$, whereas the rate constant for the stiff spring $\left(k_{\mathrm{S}} a^{2} / k_{\mathrm{B}} T=10^{4}\right)$ can be depicted by the theory with $\left(\gamma_{1}+\gamma_{0}\right)$. Furthermore, it seems reasonable to infer that $k(F)$ for the intermediate $k_{\mathrm{s}}$ regime may be described by the

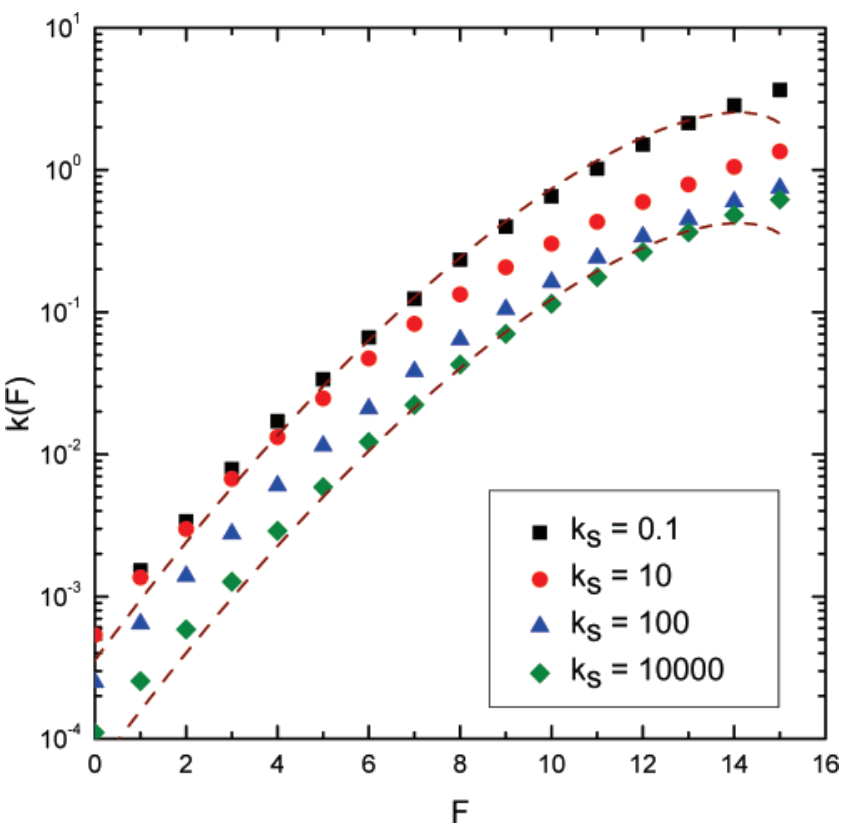

Figure 2. The variation of the rate constant with the external force is plotted for different spring constants with the critical force $\beta F_{\mathrm{c}} a=$ $5 \pi$. The dashed lines denote the prediction based on the forced Kramers rate theory, with $\gamma_{1}$ and $\left(\gamma_{0}+\gamma_{1}\right)$. The linkage stiffness $k_{\mathrm{s}}$ is scaled.

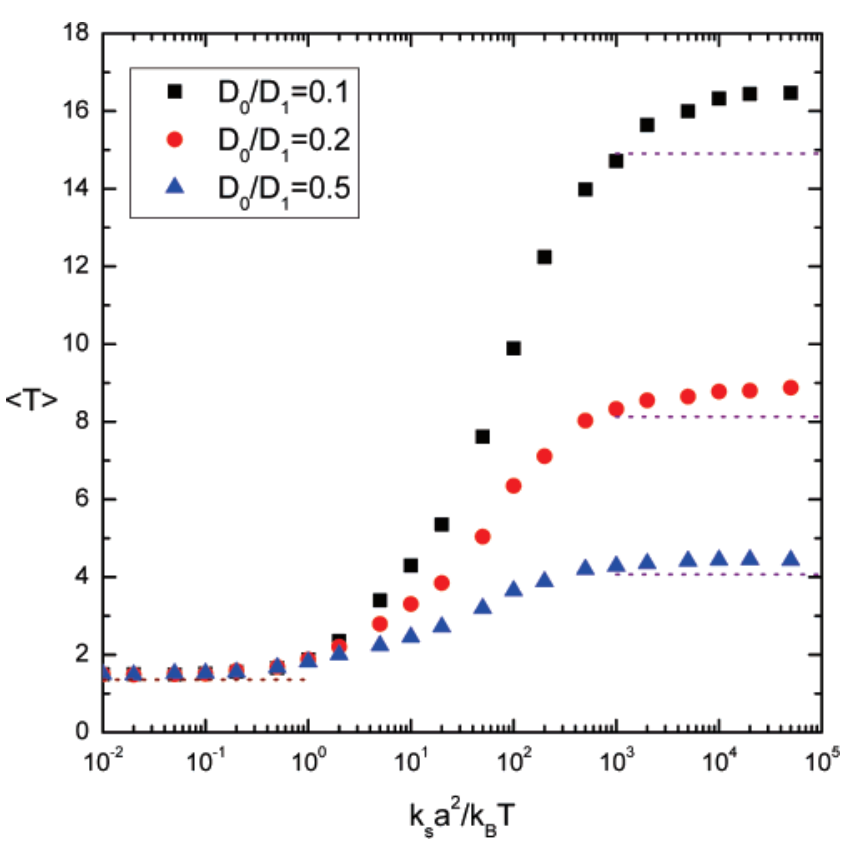

Figure 3. The variation of the bond lifetime with the linkage stiffness is plotted for different friction coefficients of the probe under external force $\beta F a=10$. The dashed lines denote the prediction based on the forced Kramers rate theory, with $\gamma_{1}$ for soft linkage and $\left(\gamma_{1}+\gamma_{0}\right)$ for stiff linkage.

Kramers theory, with an effective $\gamma$ with $\gamma_{1}<\gamma<\left(\gamma_{1}+\gamma_{0}\right)$. In other words, the leading effect of the linkage stiffness is to alter the effective friction coefficient of the ligand. As the external force decays, nevertheless, the Kramers theory underestimates the rupture rate. This result will be explained later by the secondary effect, force fluctuations.

A. Effetive Ligand Diffusivity. Figure 3 shows that variation of the mean bond lifetime $\langle T\rangle$ with the spring constant, $k_{\mathrm{s}}$, under constant pulling $\beta F a=10$. Clearly, the rupture rate declines with an increase in the linkage stiffness. In the limits of soft 


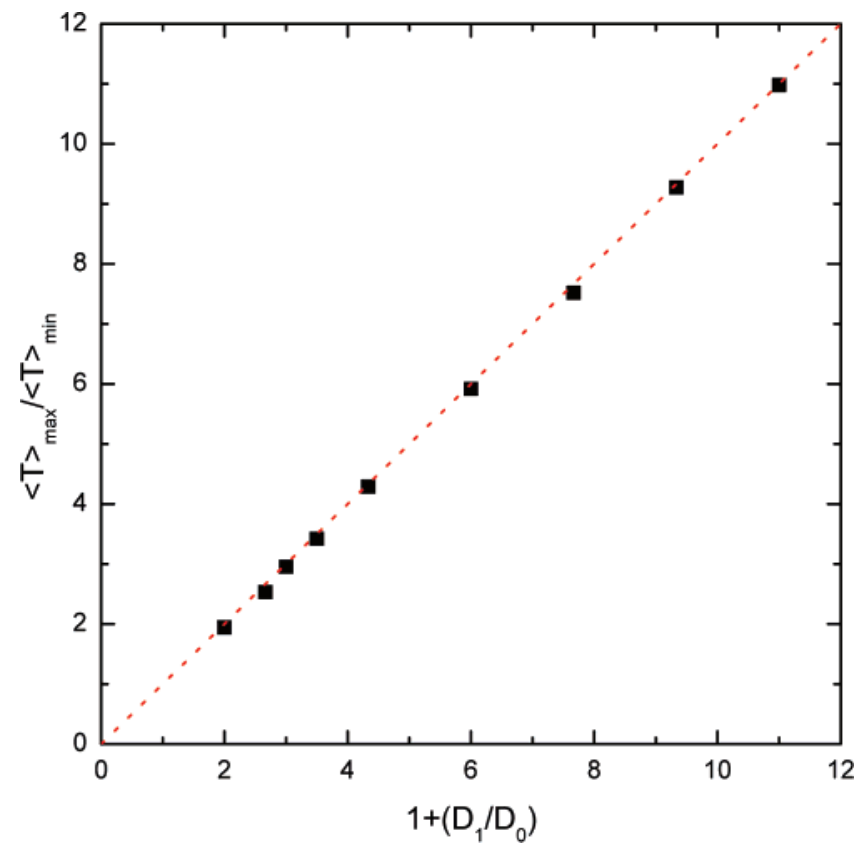

Figure 4. The bond lifetime ratio of stiff spring $\left(\langle T\rangle_{\max }\right)$ to soft spring $\left(\langle T\rangle_{\min }\right)$ is plotted against the diffusivity ratio of ligand $\left(D_{1}\right)$ to probe plus ligand $\left(D=k_{\mathrm{B}} T /\left(\gamma_{1}+\gamma_{0}\right)\right)$.

and stiff spring constants, asymptotic behavior can be seen. For $k_{\mathrm{s}} a^{2} / k_{\mathrm{B}} T \ll 1$, the mean bond lifetime is independent of the friction coefficient ratio of ligand to probe $\left(\gamma_{1} / \gamma_{0}=D_{0} / D_{1}\right)$. That is, the coupling between the ligand and probe is weak. The simulation result $\left(\langle T\rangle_{\min } \cong 1.50\right)$ agrees with the prediction by the forced Kramers rate theory associated with $\gamma_{1}$ (1.36). This consequence reveals that the rupture kinetics obtained by the soft linkage is essentially equivalent to constant pulling acting directly on the ligand. On the other hand, for $k_{\mathrm{S}} a^{2} / k_{\mathrm{B}} T \gg$ 1 , the mean bond lifetime approaches another asymptotic value, which rises with decreasing $D_{0} / D_{1}$. The increment in rupture time can be attributed to the strong coupling between ligand and probe. In fact, for a rigid linkage, the ligand and probe should be regarded as one particle. That is, the resistance to the probe must be fully accounted for during the rupture process, and the effective friction coefficient of the ligand then becomes $\left(\gamma_{1}+\gamma_{0}\right)$. As a result, one anticipates that the rupture time in the stiff spring limit $\langle T\rangle_{\max }$ corresponds to the upper limit.

$$
\langle T\rangle_{\max }=\left(\frac{\gamma_{1}+\gamma_{0}}{\gamma_{1}}\right)\langle T\rangle_{\min }
$$

Figure 4 confirms the above argument by varying $\gamma_{0} / \gamma_{1}$. Equation 18 also holds under different external forces and barrier heights.

According to the forced Kramers rate theory, the rate constant can be realized by

$$
k(F) \sim \frac{\beta E_{a}}{a^{2} / D_{\text {eff }}} \exp \left[-\beta E_{a}(F)\right]
$$

The leading order effect of linkage stiffness is to alter the behavior of ligand diffusion in the trap. The effective diffusivity of the ligand is related to its effective friction coefficient $\left(D_{\text {eff }}\right.$ $=k_{\mathrm{B}} T / \gamma_{\mathrm{eff}}$ ). For weak coupling (soft linkage), the ligand diffusivity is not influenced by the linkage, and $D_{\text {eff }} \cong D_{1}$. One is able to extract the kinetic information associated with the ligand-receptor complex. On the other hand, for strong coupling (stiff linkage), the ligand and probe are essentially combined to one trapped particle, and $D_{\text {eff }} \cong D_{1}\left(1+D_{1} / D_{0}\right)^{-1}$. Consequently, the rupture rate is reduced by $\left(1+D_{1} / D_{0}\right)^{-1}$. In the intermediate regime, the effective ligand diffusivity declines from $D_{1}$ to $D_{1}\left(1+D_{1} / D_{0}\right)^{-1}$ as the extent of coupling (the linkage stiffness $k_{\mathrm{s}}$ ) is increased. Since the major influence of $k_{\mathrm{s}}$ is to alter the pre-exponential factor, the barrier height can be reasonably extracted by constant pulling experiments, regardless of the linkage stiffness.

The reduction of the effective diffusivity can be grasped by considering the free Brownian motion of two elastically coupled particles. Basically, the mean-square displacement of the ligand $\left(x_{1}\right)$ can be obtained by solving eqs 1 and 2 in the absence of the force terms $\partial U / \partial x_{1}$ and $F$. The time evolution of the meansquare displacement of the ligand $\left\langle x_{1}^{2}\right\rangle$ can be obtained from the overdamped Langevin equation:

$$
\gamma_{1} \frac{\mathrm{d}\left\langle x_{1}^{2}\right\rangle}{\mathrm{d} t}=2 k_{\mathrm{B}} T+2 k_{\mathrm{s}}\left[\left\langle x_{1} x_{0}\right\rangle-\left\langle x_{1}^{2}\right\rangle\right]
$$

This equation clearly shows the coupling between the ligand and probe by the spring, that is, the second term on the righthand side, alters the diffusive behavior of the ligand. A similar equation can be obtained for the probe $\left\langle x_{0}{ }^{2}\right\rangle$, as well. If one defines the hydrodynamic center of resistance as

$$
x_{\mathrm{c}}=\frac{\gamma_{0} x_{0}+\gamma_{1} x_{1}}{\gamma_{0}+\gamma_{1}}
$$

then the overdamped Brownian motion of the center becomes

$$
\left(\gamma_{0}+\gamma_{1}\right) \frac{\mathrm{d} x_{\mathrm{c}}}{\mathrm{d} t}=R_{1}(t)+R_{2}(t)
$$

This equation shows that the mean-square displacement of the center follows

$$
\left\langle x_{\mathrm{c}}^{2}\right\rangle=2 \frac{k_{\mathrm{B}} T}{\gamma_{0}+\gamma_{1}} t
$$

Thus, the diffusivity of the resistance center is $D_{1}\left(1+D_{1} / D_{0}\right)^{-1}$. Using eqs 20 and 22 , the correlation term $\left\langle x_{0} x_{1}\right\rangle$ can be expressed in terms of $\left\langle x_{1}{ }^{2}\right\rangle$ and $\left\langle x_{0}{ }^{2}\right\rangle$. Solving the equations for $\left\langle x_{1}{ }^{2}\right\rangle$ and $\left\langle x_{0}{ }^{2}\right\rangle$ simultaneously gives the time-dependent behavior of the mean-square displacement,

$\left\langle x_{1}{ }^{2}\right\rangle=\frac{\gamma_{\mathrm{m}}}{\left(\gamma_{0}+\gamma_{1}\right)} \frac{\gamma_{0}}{\gamma_{1}} \frac{k_{\mathrm{B}} T}{k_{\mathrm{s}}}\left[1-\exp \left(-2 \frac{k_{\mathrm{s}}}{\gamma_{\mathrm{m}}} t\right)\right]+2 \frac{k_{\mathrm{B}} T}{\gamma_{0}+\gamma_{1}} t$

where $\gamma_{\mathrm{m}}=\gamma_{0} \gamma_{1} /\left(\gamma_{0}+\gamma_{1}\right) .\left\langle x_{0}^{2}\right\rangle$ can be obtained by exchanging the subscript 0 with 1 in eq 23 . The solution satisfies the equipartition theorem,

$$
\left\langle\left(x_{0}-x_{1}\right)^{2}\right\rangle=\frac{k_{\mathrm{B}} T}{k_{\mathrm{s}}}
$$

where we assume $l=0$.

One can define the effective diffusivity of the ligand as

$$
D_{\text {eff }}=\frac{1}{2} \frac{\mathrm{d}\left\langle x_{1}^{2}\right\rangle}{\mathrm{d} t}=\frac{k_{\mathrm{B}} T}{\gamma_{0}+\gamma_{1}}\left[1+\frac{\gamma_{0}}{\gamma_{1}} \exp \left(-2 \frac{k_{\mathrm{s}}}{\gamma_{\mathrm{m}}} t\right)\right]
$$

Note that the diffusive behavior of the ligand evolves over time, even in the absence of external pulling. As $t \ll \gamma_{\mathrm{m}} / 2 k_{\mathrm{s}}$, one has 


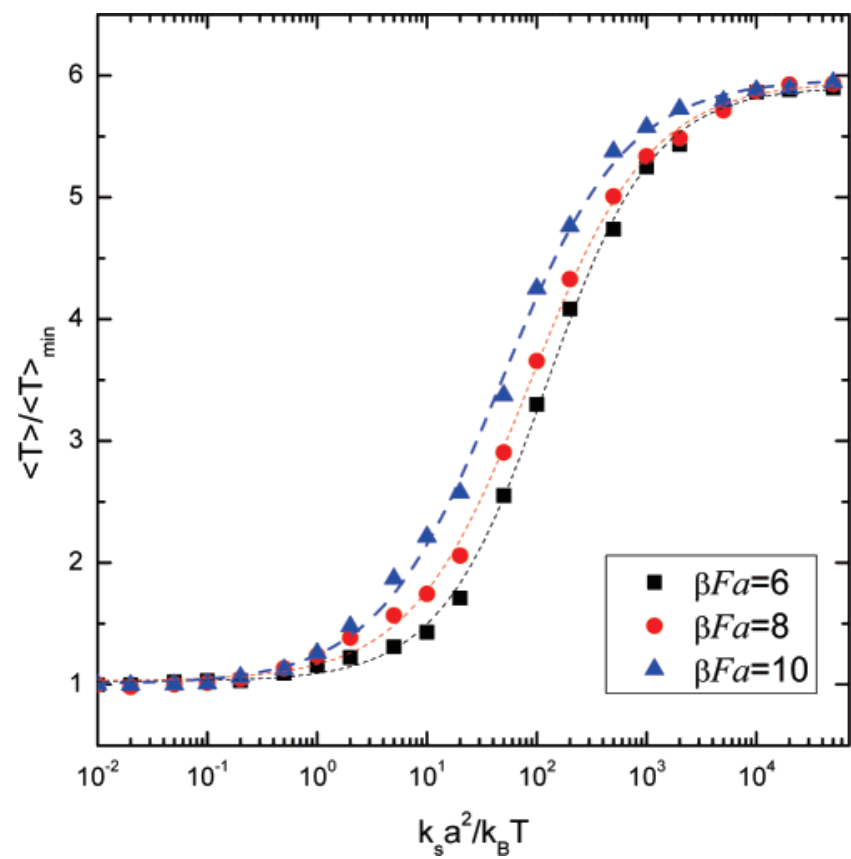

Figure 5. The variation of the bond lifetime $\langle T\rangle /\langle T\rangle_{\min }$ with the spring constant is plotted for various external forces with $D_{0} / D_{1}=0.2$. The lines are drawn to guide the eye.

$\left\langle x_{1} x_{0}\right\rangle \cong 0$ and $D_{\text {eff }} \approx k_{\mathrm{B}} T / \gamma_{1}$. This consequence reveals that the short-time diffusive behavior of the ligand can be described by the diffusivity associated with a single ligand. As time proceeds, the effective diffusivity declines. Although the diffusive behavior of the ligand approaches the diffusivity of the resistance center $\left(x_{c}\right)$ eventually, $D_{\text {eff }} \approx k_{\mathrm{B}} T /\left(\gamma_{1}+\gamma_{0}\right)$, it will take place only when $t \gg \gamma_{\mathrm{m}} / 2 k_{\mathrm{s}}$. Evidently, the influence of the linkage stiffness is manifested through the characteristic time associated with the diffusivity crossover $\left(\tau_{\mathrm{r}}=\gamma_{\mathrm{m}} / 2 k_{\mathrm{s}}\right)$. For a soft linkage, the bond lifetime is short compared to $\tau_{\mathrm{r}}$, that is, $\langle T\rangle \ll \gamma_{\mathrm{m}} / 2 k_{\mathrm{s}}$, and thus, the rupture kinetics is controlled by the ligand alone. On the contrary, for a stiff linkage, the bond lifetime is long compared to $\tau_{\mathrm{r}}$, that is, $\langle T\rangle \gg \gamma_{\mathrm{m}} / 2 k_{\mathrm{s}}$, and therefore, the rupture kinetics is determined by both ligand and probe.

B. Force Fluctuations. If the effect of the linkage stiffness is simply to change the effective ligand diffusivity $D_{\text {eff }}$, then one would expect that $\langle T\rangle /\langle T\rangle_{\min }$ is independent of external force $F$. That is, the variation of $\langle T\rangle /\langle T\rangle_{\min }$ with $k_{\mathrm{s}}$ for different $F \mathrm{~s}$ can be represented by a single curve. As shown in Figure 5, $\langle T\rangle /\langle T\rangle_{\min }$ is independent of $F$ in the two asymptotic limits. However, in the intermediate regime, $\langle T\rangle\left\langle\langle T\rangle_{\min }\right.$ is increased with $F$. This consequence discloses the fact that the secondary effect, spring force fluctuations, may come into play. The force fluctuation is caused by thermal motions associated with ligand and probe. Since the spring cannot respond to the thermal fluctuations instantaneously, the extension of the spring is not maintained at a fixed length, corresponding to constant external force. Consequently, the ligand feels a time-fluctuating pulling force.

The spring force fluctuation can be understood by the distribution of fluctuating forces. Figure $6 \mathrm{a}-\mathrm{c}$ demonstrates the typical distribution of the spring force acting on the ligand. Under a strong external force, $F a / k_{\mathrm{B}} T=10$, the standard deviation is small $\left(\sigma_{F}=7.3 \times 10^{-3}\right)$ for a soft spring, $k_{\mathrm{s}} a^{2} / k_{\mathrm{B}} T$ $=0.01$, as show in Figure 6a. This result confirms that the constant pulling condition can be achieved as $F / k_{\mathrm{s}} a \gg 1$. However, for a stiff spring, $k_{\mathrm{s}} a^{2} / k_{\mathrm{B}} T=100$, the force distribution
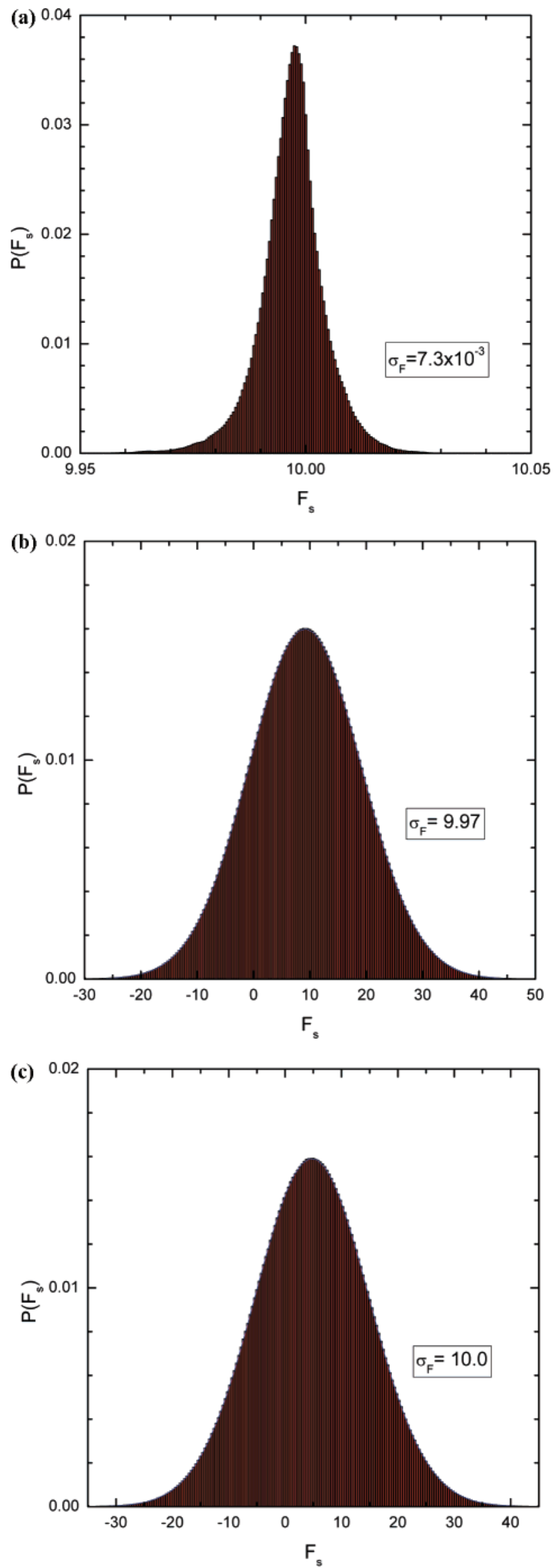

Figure 6. The typical spring force distributions $P\left(F_{\mathrm{s}}\right)$. The spring force, $F_{\mathrm{s}}$, is scaled by $k_{\mathrm{B}} T / a$. (a) Soft spring, $k_{\mathrm{s}} a^{2} / k_{\mathrm{B}} T=10^{-2}$ under $\beta F a=$ 10 ; (b) stiff spring, $k_{\mathrm{s}} a^{2} / k_{\mathrm{B}} T=10^{2}$ under $\beta F a=10$; and (c) stiff spring, $k_{\mathrm{S}} a^{2} / k_{\mathrm{B}} T=10^{2}$ under $\beta F a=5$. 


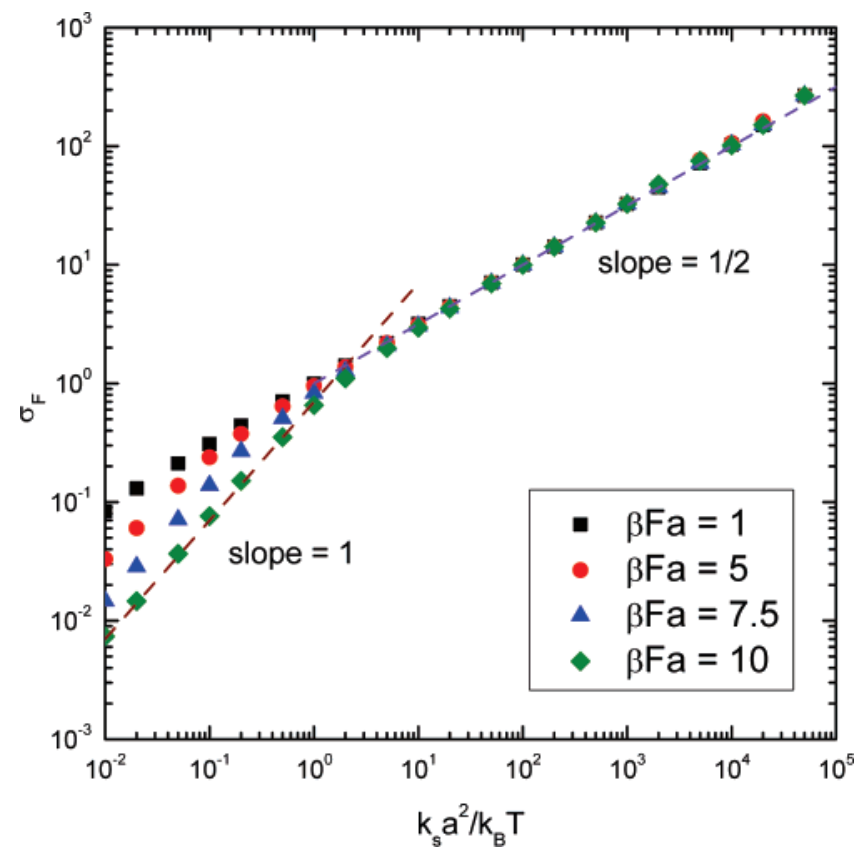

Figure 7. The variation of the standard deviation of the force fluctuation with the spring constant is plotted for various external forces. The dashed lines denotes $\sigma_{F} \propto \sqrt{k_{\mathrm{s}}}($ slope $=1 / 2)$ and $\sigma_{F} \propto k_{\mathrm{S}}$ (slope $=1)$.

is Gaussian-like, and its standard deviation becomes large $\left(\sigma_{F}\right.$ $=9.97$ ), as depicted in Figure 6b. This outcome reveals that the spring force may fluctuate substantially from about $F-$ $2 \sigma_{F}$ to $F+2 \sigma_{F}$, and the force acting directly on the ligand is not constant at all. Using the same spring but under weaker external force, $F a / k_{\mathrm{B}} T=5$, the standard deviation is essentially the same $\left(\sigma_{F}=10.0\right)$, as illustrated in Figure $6 \mathrm{c}$. It seems that the distribution of the spring force is sensitive to the spring stiffness, $k_{\mathrm{s}}$, but not the external force, $F$. The above analysis for free Brownian motion of two elastically coupled particles may help us to clarify this point.

The standard deviation of the spring force fluctuation may be estimated by the equipartition theorem for $\langle T\rangle \gg \gamma_{\mathrm{m}} / 2 k_{\mathrm{s}}$ :

$$
\sigma_{F} \cong k_{\mathrm{s}}\left\langle\left(x_{0}-x_{1}-l\right)^{2}\right\rangle^{1 / 2}=\left(k_{\mathrm{s}} k_{\mathrm{B}} T\right)^{1 / 2}
$$

With a specified $\langle T\rangle$ (or a given $F$ ), the standard deviation has to follow eq 26 for large enough $k_{\mathrm{s}}$. On the other hand, for a given $k_{\mathrm{s}}$, eq 26 is valid for small enough $F$ because $\langle T\rangle$ declines with increasing $F$. For a stiff spring, the condition $\langle T\rangle \gg \gamma_{\mathrm{m}} /$ $2 k_{\mathrm{S}}$ is generally valid, and therefore, the distribution of the fluctuating force is essentially force-independent. For a soft spring under strong pulling, however, the condition $\langle T\rangle \gg \gamma_{\mathrm{m}}$ ' $2 k_{\mathrm{s}}$ cannot be satisfied, and the standard deviation may become external-force-dependent. The variation of $\sigma_{F}$ with $k_{\mathrm{s}}$ for different $F \mathrm{~s}$ is shown in Figure 7. Evidently, $\sigma_{F}$ is forceindependent for a large $k_{\mathrm{s}}$ but becomes force-dependent for a small $k_{\mathrm{s}}$. Under $F a / k_{\mathrm{B}} T=1$, eq 26 is obeyed $\left(\sigma_{F} \propto \sqrt{k_{\mathrm{s}}}\right)$ for a wide range, $k_{\mathrm{s}} a^{2} / k_{\mathrm{B}} T=10^{-2} \sim 10^{5}$. Under $F a / k_{\mathrm{B}} T=10$, eq 26 is followed only when $k_{\mathrm{S}} a^{2} / k_{\mathrm{B}} T>10$. It is anticipated that the force fluctuation is proportional to $k_{\mathrm{s}} a$ for a soft spring under strong pulling because the extension fluctuation $\left(x_{0}-x_{1}\right)$ comes mainly from the random motion of ligand in the energy well. Consequently, the standard deviation for $F a / k_{\mathrm{B}} T=10$ comes to agree with the relation $\sigma_{F} \propto k_{\mathrm{s}}$ as $k_{\mathrm{s}} a^{2} / k_{\mathrm{B}} T<1$.

The effect of force fluctuation can be realized by considering the simplest case: weak pulling. When the external force is weak, adopting Bell's expression to describe the force-assisted rupture is justified. For $F a / k_{\mathrm{B}} T \lesssim 1$, the average rate constant is given by

$$
\begin{aligned}
\langle k[F(t)]\rangle & \cong\left\langle\frac{1}{\tau_{0}} \exp \left[-\beta E_{a}{ }^{0}+\beta F(t) a\right]\right\rangle \\
& =k(F=0)\langle\exp \beta F(t) a\rangle
\end{aligned}
$$

Employing the inequality relation $\langle\exp X\rangle \geq \exp \langle X\rangle$ leads to the conclusion that the fluctuations of the pulling force speed up the rupture process. Expanding the exponential, one gets

$$
\begin{aligned}
&\langle\exp X\rangle \simeq\langle X\rangle+\frac{1}{2 !}\left(\left\langle X^{2}\right\rangle-\langle X\rangle^{2}\right)+ \\
& \frac{1}{3 !}\left(\left\langle X^{3}\right\rangle-3\left\langle X^{2}\right\rangle\langle X\rangle+2\langle X\rangle^{3}\right)+\ldots
\end{aligned}
$$

Truncating the cumulant expansion of $\langle k\rangle$ to second order leads to

$$
\langle k(t)\rangle \simeq k(F=0) \exp \left[\beta\langle F\rangle a+\frac{(\beta\langle F\rangle a)^{2}}{2}\left(\frac{\left\langle F^{2}\right\rangle}{\langle F\rangle^{2}}-1\right)\right]
$$

This consequence reveals that the greater the extent of force fluctuation $\left\langle F^{2}\right\rangle$, the faster the dissociation process.

The linkage stiffness alters the effective ligand diffusivity and causes the force fluctuation acting on the ligand. The aforementioned analysis for the influence of force fluctuations neglects the change in diffusivity. In fact, the leading order effect of increasing $k_{\mathrm{S}}$ is to reduce the effective ligand diffusivity, which results in an increase in the bond lifetime. According to eq 26 , however, increasing $k_{\mathrm{s}}$ also enhances the force fluctuation, which lowers the bond lifetime. It can be regarded as the secondorder effect. The net result leads to the external-force-dependent behavior of $\langle T\rangle$ in the intermediate $k_{\mathrm{s}}$ regime, as demonstrated in Figure 5 . In this regime, $\sigma_{F}$ varies primarily with $k_{\mathrm{s}}$ and, thus, is insensitive to $F$. Since the incremental extent of the rupture rate $(\bar{k} / k)$ for the same $\sigma_{F}$ declines with increasing $F$, the relative bond lifetime $\left(\langle T\rangle /\langle T\rangle_{\min }\right)$ grows with $F$ for the same linkage stiffness.

C. Force Correlation Time and Violation of Adiabatic Condition. Force fluctuations enhance the rupture rate. Since the force fluctuation is proportional to the square root of the spring constant, as depicted in Figure 7, one expects that the effect of force fluctuation might overcome the effect of effective ligand diffusivity eventually by increasing $k_{\mathrm{s}}$. However, as shown in Figure 3, the rupture rate approaches an asymptote for stiff springs, which can be estimated solely by the effective ligand diffusivity. Evidently, the contribution associated with force fluctuations disappears at large $k_{\mathrm{s}}$. A possible explanation is that the relaxation time of force fluctuations may be too short to be followed by the ligand during the dissociation process. That is, the adiabatic approximation fails.

For a finite bond lifetime, a large $\sigma_{F}$ associated with a specified $F$ reveals that the spring force acting on the ligand fluctuates very frequently. The fluctuating forces are only correlated within a short time interval. The characteristic correlation time can be extracted from the time-correlation function, which is defined as

$$
C(t)=\frac{\langle F(0) F(t)\rangle}{\left\langle F(0)^{2}\right\rangle} \sim A \exp \left(-\frac{t}{\tau_{F, 1}}\right)+B \exp \left(-\frac{t}{\tau_{F, 2}}\right)
$$

Figure 8 shows the time-correlation functions for different linkage stiffnesses under external force $F a / k_{\mathrm{B}} T=1$. Evidently, the correlation time declines with increasing $k_{\mathrm{s}}$. The time- 


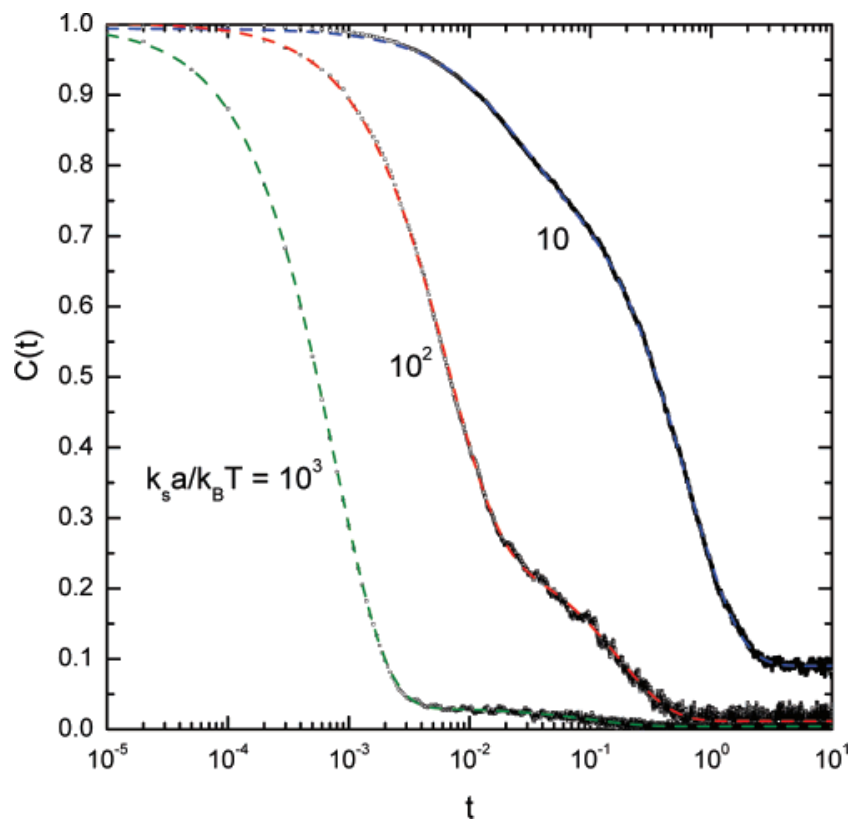

Figure 8. The time-correlation function for the fluctuating forces is plotted for different spring constants under external force $\beta F a=1$. The data points denote the simulation results, and the dashed lines represent the second-order exponential decay.

correlation function obtained from simulations can be wellrepresented by a second-order exponential decay. If the fast mode corresponds to the position fluctuation of the ligand, the correlation time $\left(\tau_{F, 1}\right)$ is anticipated to be proportional to $\gamma_{\mathrm{m}} / k_{\mathrm{s}}$. The fitting results give $\tau_{F, 1}=2.1 \times 10^{-2}, 6.5 \times 10^{-3}$, and 7.6 $\times 10^{-4}$ for $k_{\mathrm{S}} a^{2} / k_{\mathrm{B}} T=10,10^{2}$, and $10^{3}$, respectively. This result confirms that the correlation time associated with the fluctuating force is very short for stiff springs. In our simulations, however, the integration time step always satisfies the condition, $\Delta t \ll \tau_{F, 1}$.

The forced Kramers rate theory is valid when the adiabatic approximation is justified. That is, the Brownian particles are adjusted to the apparent potential (intrinsic potential plus the spring potential) rapidly. This is justified when the escape time $\langle T\rangle$ is large as compared to the local relaxation time, $\tau_{\mathrm{R}}$, in the absence of driving, where $\tau_{\mathrm{R}} \simeq \tau_{0} \approx\left(a^{2} / D\right) / \beta E_{a}{ }^{0}$. However, as the spring potential varies too fast, that is, $\tau_{\mathrm{R}} \gg \tau_{F}$, the ligand is unable to follow its instant change. In the present study, $\tau_{\mathrm{R}}$ $\sim O(0.1) a^{2} / D$. For springs with stiff enough linkage, the force correlation time is short compared to the local relaxation time. For example, $\tau_{F} \sim O\left(10^{-3}\right) a^{2} / D$ for $k_{\mathrm{s}} a^{2} / k_{\mathrm{B}} T=10^{3}$. As a consequence, the adiabatic approximation is not satisfied, and the force fluctuation becomes unimportant. Nonetheless, the ligand can still feel the average force $\langle F\rangle$, and hence, the rupture kinetics for stiff linkage follows the forced Kramers rate theory under constant force condition, even though the fluctuating force is a few times the average force.

\section{Conclusion}

In single-molecule pulling experiments, the bonded region (ligand) is actually linked to the force-acting region (probe) by an elastic linkage. The stiffness of the linkage may affect the rupture process. By using overdamped Langevin dynamics, we investigate the kinetics of two elastically coupled Brownian particles escaping from a trap. The linkage stiffness causes three significant effects: effective ligand diffusivity, force fluctuations, and violation of adiabatic condition. The leading effect is the increment in the effective ligand diffusivity due to its coupling to the probe. For weak coupling (soft linkage), the effect associated with spring and probe can be ignored, and the true rupture rate can be extracted. On the other hand, for strong coupling (stiff linkage), the diffusivity of the probe has to be accounted for and, thus, leads to a smaller rupture rate, dependent on the diffusivity ratio between probe and ligand.

The random motion of ligand and probe causes the fluctuation of the spring force acting on the ligand. Compared with the rupture rate associated with a constant force $k(F)$, the bond lifetime is reduced by force fluctuation around $F$. The rupture rate increased by the fluctuating force greater than $F$ is more than that impeded by the force less than $F$. Generally, the standard deviation of the fluctuating force distribution can be estimated by the equipartition theorem and, hence, is proportional to the linkage stiffness. As a result, force fluctuations retard the rise in the bond lifetime as the ligand-probe coupling is increased. For stiff springs, however, the fluctuating force is too fast to be followed by the ligand during the rupture process. That is, the adiabatic condition is violated. Since the force fluctuation becomes unimportant, the ligand feels the average force only.

Acknowledgment. This work is supported by the National Science Council of Taiwan under grant number NSC 94-2214E-002-020 (H.J.L. and Y.J.S.), NSC 94-2112-M-008-020 (H.Y.C.), and NSC 94-2214-E-008-001 (H.K.T.).

\section{References and Notes}

(1) Evans, E. Annu. Rev. Biophys. Biomol. Struct. 2001, 30, 105

(2) Florin, E. L.; Moy, V. T.; Gaub, H. E. Science 1994, 264, 415

(3) Hummer, G.; Szabo, A. Biophys. J. 2003, 85, 5.

(4) Dudko, O. K.; Filippov, A. E.; Klafter, J.; Urbakh, M. Proc. Natl. Acad. Sci. U.S.A. 2003, 100, 11378.

(5) Chen, H.-Y.; Chu, Y.-P. Phys. Rev. E: Stat. Phys., Plasmas, Fluids, Relat. Interdiscip. Top. 2005, 71, 010901(R).

(6) Sheng, Y.-J.; Jiang, S.; Tsao, H.-K. J. Chem. Phys. 2005, 123, 091102 .

(7) Zhou, J.; Zhang, L.; Leng, Y.; Tsao, H.-K.; Sheng, Y.-J.; Jiang, S. J. Chem. Phys. 2006, 125, 104905.

(8) Evans, E.; Ritchie, K. Biophys. J. 1999, 76, 2439.

(9) Sain, A.; Wortis, M. Phys. Rev. E: Stat. Phys., Plasmas, Fluids, Relat. Interdiscip. Top. 2004, 70, 031102.

(10) Clarke, R. J.; Jensen, O. E.; Billingham, J.; Pearson, A. P.; Williams, P. M. Phys. Rev. Lett. 2006, 96, 050801.

(11) Kramers, H. A. Physica (Amsterdam) 1940, 7, 284

(12) Hänggi, P.; Talkner, P.; Borkovec, M. Rev. Mod. Phys. 1990, 62, 251.

(13) Bell, G. I. Science 1978, 200, 618. 15592

(14) Garg, A. Phys. Rev. B: Condens. Matter Mater. Phys. 1995, 51, (15)

(15) Lin, H.-J.; Chen, H.-Y.; Sheng, Y.-J.; Tsao, H.-K. Phys. Rev. Lett. 2007, 98, 088304 . 